\title{
Psoriasis beyond the skin: Ophthalmological changes (Review)
}

\author{
MARIA-MAGDALENA CONSTANTIN ${ }^{1,2}$, MARIA-DIANA CIURDUC ${ }^{2}$, STEFANA BUCUR $^{1,2}$, \\ RODICA OLTEANU ${ }^{2}$, RAZVAN ADRIAN IONESCU ${ }^{1,3}$, \\ TRAIAN CONSTANTIN ${ }^{1,4}$ and FLORENTINA FURTUNESCU ${ }^{1,5}$
}

\author{
${ }^{1}$ Third Department, 'Carol Davila' University of Medicine and Pharmacy, 050474 Bucharest; \\ ${ }^{2}$ Second Department of Dermatology and ${ }^{3}$ Third Department of Internal Medicine and Rheumatology, \\ Colentina Clinical Hospital, 020125 Bucharest; ${ }^{4}$ Department of Urology, 'Prof. Dr. Th. Burghele' Hospital, 050659 Bucharest; \\ ${ }^{5}$ Department of Public Health and Management, National Institute of Public Health, 050463 Bucharest, Romania
}

Received February 27, 2021; Accepted June 3, 2021

DOI: $10.3892 / \mathrm{etm} .2021 .10413$

\begin{abstract}
Psoriasis is a chronic, immune-mediated inflammatory disease of unknown etiology that may be associated with abnormal T-lymphocyte function. Ocular manifestations associated with psoriasis, particularly artropathic or pustular psoriasis, usually affect men, often during exacerbations of the disease. It has been reported that eye damage tends to occur later compared with cutaneous or joint manifestations, blindness being the most disabling complication. Previous studies have focused on ophthalmic manifestations and identified several etiopathogenic mechanisms. Psoriasis may be associated with eye complications such as lesions of the eyelids, conjunctiva and others, with systemic inflammation being the main contributor. In addition, the treatment used for psoriasis may cause ocular changes. The main ophthalmic manifestations associated with psoriasis are keratoconjunctivitis sicca, blepharitis, conjunctivitis and uveitis. The treatment of uveitis, perceived as one of the most serious eye conditions, is controversial and has yet to be clearly determined. Thus, the aim of the present review was to emphasize the importance of regular eye examination for patients with psoriasis, either those receiving biological treatment or those not receiving treatment, in order to diagnose and manage the disease appropriately.
\end{abstract}

\section{Contents}

1. Introduction

2. Etiopathogenesis

3. Ophthalmological changes

Correspondence to: Dr Maria-Diana Ciurduc, Second Department of Dermatology, Colentina Clinical Hospital, 19-21 Stefan cel Mare Blvd, 020125 Bucharest, Romania

E-mail:diana.ciurduc@yahoo.com

Key words: psoriasis, ocular changes, uveitis, biologics
4. Future directions

5. Conclusions

\section{Introduction}

Psoriasis is a chronic, immune-mediated disease with high genetic susceptibility and significant multisystem damage. Psoriasis affects $1-3 \%$ of the adult population, has a negative impact on the quality of life of the patients (1), and it is no longer considered as a disease exclusively affecting the skin, as it is associated with several systemic complications, including cardiovascular, metabolic, cerebrovascular, articular, hepatic, autoimmune and ocular damage. Currently, ophthalmological manifestations have an occurrence rate of $10-12 \%$ among patients with psoriasis $(2,3)$. However, these figures do not reflect the true magnitude of how significantly this organ is affected.

\section{Etiopathogenesis}

From an embryological point of view, the skin and eyes share a common origin from the ectoderm (4). Thus, it is hypothesized that the ocular manifestations are closely associated with the cutaneous manifestations through an immune-mediated mechanism. Skin histology appears to be similar to ocular histology (parakeratosis), particularly in cases where psoriatic lesions are observed at the level of the eyelids or the conjunctiva $(5,6)$.

Eye changes in patients with psoriasis are considered to be a consequence of systemic inflammation (7) and increased cytokine production; however, they are often overlooked, although their progression may result in visual impairment. Changes in the tear film, the conjunctival surface, or disruption of the meibomian glands are common among patients with psoriasis.

In addition to psoriasis per se being the causative factor, current treatment strategies, including methotrexate and acitretin (the only currently licensed drug for generalized pustular psoriasis) may cause ocular damage; during phototherapy (a valuable and cost-effective anti-psoriatic treatment), UVA rays may penetrate into the ocular lens and potentially induce cataract; furthermore, biological therapies, such as anti-TNF $\alpha$, may also cause ocular manifestations, such as especially uveitis, 
in psoriatic patients (8). Since 1988, it has been well-known that treatment with retinoids, including isotretinoin, affects almost all anatomical structures of the eye. Notable injuries are commonly observed in the optic nerve, retina or lens (9-11). Furthermore, there are several changes to the eyes, potentially affecting almost any structural region, which are associated with arthropathic or pustular psoriasis (5).

\section{Ophthalmological changes}

The most common clinical ocular change associated with psoriasis is keratoconjunctivitis sicca (dry eye syndrome), which is present in $18.75 \%$ of patients, and blepharitis $(2,12,13)$. Other changes include conjunctivitis, uveitis, punctate keratitis, pinguecula, cataracts, glaucoma, corneal abscesses, pterygium, or abnormalities of retinal vascularization $(5,14)$. An uncommon condition that may also develop is pigment dispersion syndrome, which is characterized by shedding of the pigment from the posterior surface of the iris into the anterior segment following the flow of aqueous humour.

Several studies on patients with psoriasis have demonstrated an association between Psoriasis Area and Severity Index (PASI) scores and the presence of ocular manifestations. Patients with blepharitis tend to have low PASI scores, whilst patients with keratitis have moderate-to-severe PASI scores $(2,14)$. Paradoxically, a study performed in 2013 on 100 patients with psoriasis concluded that there is no significant association between severe cases of psoriasis and eye lesions; therefore, all patients should be evaluated ophthalmologically to establish an accurate diagnosis (12).

Keratoconjunctivitis sicca. Dry eye syndrome occurs in patients with psoriasis due to an obstructive dysfunction of the excretory ducts of the meibomian glands. Although their secretory function is normal, the dysfunction results from epithelial keratinization of the glandular ducts $(1,2)$. The patients experience a feeling of dry eyes, foreign body sensation, blurry vision and photophobia, with symptoms worsening throughout the day (6). This complication may be independent from or may be the result of blepharitis or conjunctivitis. Conversely, this syndrome may be associated with the decrease of the tear film, namely the deficiency of L-arginine, which is observed in patients with psoriasis (15).

Blepharitis. Blepharitis is one of the most common ocular manifestations associated with psoriasis. Patients exhibit hyperemia, inflammation and edema at the free margin of the eyelids, with slight scaling, itching and burning sensation $(1,6,13)$. Chronic blepharitis may lead to ectropion of the lower lacrimal point with epiphora, madarosis, trichiasis and loss of lid tissue $(5,13)$ or meibomian gland dysfunction, which is frequently associated with posterior blepharitis.

The treatment of patients with blepharitis includes rigorous hygiene (warm compresses, washing the eyelids and lashes with a gentle shampoo) and application of topical corticosteroids on the eyelids (6). The application of tacrolimus ointments may also be taken into consideration (16). In addition, antibiotic ointment application may be necessary for the treatment of secondary bacterial infections; should such complications occur surgery is performed, including in cases of ectropion, trichiasis and pterygium (13).
Table I. Uveal involvement in various autoimmune diseases ${ }^{\mathrm{a}}$.

\begin{tabular}{|c|c|c|}
\hline Autoimmune disease & Affected uveal segment & (Refs.) \\
\hline Psoriasis & Anterior & $(26)$ \\
\hline Psoriatic arthritis & Anterior & $(26,27)$ \\
\hline Behçet's disease & $\begin{array}{l}\text { Posterior and panuveitis } \\
\text { (retinal necrosis) }\end{array}$ & $(28,29)$ \\
\hline Spondyloarthritis & Anterior & $(24,29)$ \\
\hline Sarcoidosis & Anterior/posterior/panuveitis & $(30,31)$ \\
\hline $\begin{array}{l}\text { Juvenile idiopathic } \\
\text { arthritis }\end{array}$ & $\begin{array}{l}\text { Anterior-leading to chronic } \\
\text { iridocyclitis }\end{array}$ & $(27,32)$ \\
\hline
\end{tabular}

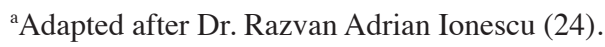

Conjunctivitis. Conjunctivitis manifests as hyperemia, possible yellowish-red lesions in the conjunctival area and xerosis that may involve the cornea (1). Patients complain of the feeling of grittiness or foreign bodies in the eyes, pain and conjunctival secretions. In cases with complications, such as trichiasis or symblepharon, patients should be referred to an ophthalmologist $(6,13)$.

Cataract. Several studies have previously reported that patients with psoriasis may develop cataracts $(8,13,14)$. Lens abnormalities, which are generally considered to be incidental findings, are defined by gradually declining vision, glare around lights, decreased contrast sensitivity and visualization of cloudy lens on routine ophthalmological examination. Ultraviolet (UV) radiation contributes to cataract development, as light in the wavelength range of $300-400 \mathrm{~nm}$ is absorbed in the lens and may cause photochemical changes in the lens proteins. It has been hypothesized that treatment with psoralen-UVA may trigger the formation of anterior cataracts, whereas prolonged courses of systemic corticosteroids for psoriasis management may cause posterior subcapsular cataracts (13).

Uveitis. Uveitis is a rare but serious eye complication that occurs in patients with psoriasis, and is the most common cause of blindness in developed countries. Anterior uveitis affects the iris, cornea and ciliary body, intermediate uveitis affects the vitreous body and pars plana, and posterior uveitis affects the retina. Anterior uveitis is more common in patients with psoriasis, and it is usually diagnosed at an early stage. Several studies have reported the occurrence of anterior uveitis in 7-25\% of patients with psoriasis $(1,17,18)$. This is a bilateral condition and the symptoms include red, painful eyes with pericorneal congestion, reduction or even loss of visual acuity and photophobia may also occur $(1,6,12,19)$. Previous studies have demonstrated the association between uveitis and different types of psoriasis, but uveitis is more frequent among patients with HLA B27-positive psoriatic arthritis (20); however, the association is not fully understood. Uveitis may also develop in patients with pustular psoriasis $(5,12)$. Uveitis is not only a complication of psoriasis, but also of other immune-mediated diseases. It affects several anatomical structures of the eye, without the involvement of any infectious factors (Table I) (14,21-32). This commonly results in disabling complications, such as cataract, glaucoma, band keratopathy or macular edema $(23,25)$. 
Corticosteroids are commonly used to treat uveitis, followed by immunosuppressive treatments. In patients who do not respond to these therapeutic options, therapy with $\mathrm{TNF} \alpha$ inhibitors (anti-TNF $\alpha$ ) may be considered $(1,17,21)$. Although the majority of studies regarding anti-TNF $\alpha$ treatment have demonstrated promising results in terms of the remission of ocular inflammation, cases of exacerbation or eye damage have also been reported. For example, a case reported in 2015 described the induction of uveitis in a patient with ulcerative colitis treated with infliximab (33). Another study demonstrated that $15 / 54$ patients treated at least once with anti-TNF $\alpha$ developed anterior uveitis following therapy. If the treatment is not effective, two possibilities may be considered: TNF $\alpha$ inhibitors are not well-tolerated and do not achieve an appropriate therapeutic response in ocular damage, or $\mathrm{TNF} \alpha$ inhibitors induce uveitis (34). Conversely, a recent article demonstrated the efficacy of certolizumab pegol in cases of refractory uveitis or in patients who do not tolerate infliximab or adalimumab (35).

Psoriasis and uveitis have similar immune etiopathogenetic mechanisms, as a result of immunological disturbances in the Th1 and Th17 lymphocyte populations. T17 cells play a key role in sustaining the inflammatory cascade in psoriasis. Secukinumab was the first IL-17A inhibitor to emerge as one of the preferred biological agents to manage chronic psoriasis in patients with multiple comorbidities, including uveitis (36). Notably, ustekinumab has also demonstrated good results in uveitis (37). The incidence of anterior uveitis during treatment with ixekizumab was described; however, in several cases, patients had a pre-existing history of anterior uveitis and were HLA B27 $7^{+}(38)$.

Therefore, although biological therapies are increasingly used for the treatment of moderate-to-severe psoriasis, their ocular safety profiles remain unclear and further investigation is required.

\section{Future directions}

Dermatologists, rheumatologists and ophthalmologists must be vigilant when questioning and examining patients with psoriasis, and a multidisciplinary approach must be employed to diagnose ocular psoriasis early to manage unnecessary discomfort, minimize eye damage and prevent potential permanent loss of vision. Ocular manifestations of psoriasis may be subtle and may be missed in the absence of a dedicated ophthalmological examination at regular intervals.

Understanding the critical checkpoints in the pathogenesis of ocular changes is important for providing the clinician with relevant targets for immunotherapeutic intervention. Therapies have been moving away from general immunosuppression toward the use of specific biologicals, targeting defined aspects of the immune response.

\section{Conclusion}

Ophthalmic complications of psoriasis are common and can affect almost any part of the eye. However, they remain clinically underappreciated. Routine eye examinations are recommended in patients with all types of psoriasis. Periodic ophthalmological evaluation and monitoring are required for early diagnosis and treatment, regardless of the severity and duration of the disease, or the presence of nail or joint lesions, in order to avoid irreversible ocular damage. Undetected ocular involvement, if left undiagnosed in the early stages, may progress asymptomatically, ultimately becoming irreversible. In this context, it is crucial to include an experienced ophthalmologist in a multidisciplinary team that has experience with treating patients with multiple comorbidities.

\section{Acknowledgements}

Not applicable.

\section{Funding}

No funding was received.

\section{Availability of data and materials}

Not applicable.

\section{Authors' contributions}

MMC, MDC, SB, RO, RAI, TC and FF contributed equally to the acquisition, analysis and systematization of data, manuscript writing and critical revision for important intellectual content. All the authors have read and approved the final version of the manuscript.

\section{Ethics approval and consent to participate}

Not applicable.

\section{Patient consent for publication}

Not applicable.

\section{Competing interests}

The authors declare that they have no competing interests.

\section{References}

1. Rajguru JP, Maya D, Kumar D, Suri P, Bhardwaj S and Patel ND: Update on psoriasis: A review. J Family Med Prim Care 9: 20-24, 2020.

2. Cruz NFSD, Brandão LS, Cruz SFSD, Cruz SASD, Pires CAA and Carneiro FRO: Ocular manifestations of psoriasis. Arq Bras Oftalmol 81: 219-225, 2018.

3. Bolognia JL, Schaffer JV and Cerroni L: Dermatology. 4th edition. Elsevier, London, pp141-149, 2018.

4. Al Akrash LS, Al Semari MA and Al Harithy R: Ocular manifestations of dermatological diseases part I: Infectious and inflammatory disorders. Int J Dermatol 60: 5-11, 2021.

5. Demerdjieva Z, Mazhdrakova I and Tsankov N: Ocular changes in patients with psoriasis. Clin Dermatol 37: 663-667, 2019.

6. Rehal B, Modjtahedi BS, Morse LS, Schwab IR and Maibach HI: Ocular psoriasis. J Am Acad Dermatol 65: 1202-1212, 2011.

7. Chimenti MS, Triggianese P, Salandri G, Conigliaro P, Canofari C, Caso F, Costa L, Nucci C, Aiello F, Cesareo M and Perricone R: A multimodal eye assessment in psoriatic arthritis patients sine-psoriasis: Evidence for a potential association with systemic inflammation. J Clin Med 9: 719, 2020.

8. Ghalamkarpour F, Baradaran-Rafii A, Sadoughi MM, Abdollahimajd F, Younespour S, Zargari O and Rudolph RI: Ocular findings in patients with psoriasis: Is it related to the side effects of treatment or to psoriasis itself? A case-control study. J Dermatolog Treat 31: 27-32, 2020. 
9. Lerman S: Ocular side effects of accutane therapy. Lens Eye Toxic Res 9: 429-438, 1992.

10. Fraunfelder FW: Ocular side effects associated with isotretinoin. Drugs Today (Barc) 40: 23-27, 2004.

11. Lebowitz MA and Berson DS: Ocular effects of oral retinoids. J Am Acad Dermatol 19: 209-211, 1988.

12. Kilic B, Dogan U, Parlak AH, Goksugur N, Polat M, Serin D and Ozmen S: Ocular findings in patients with psoriasis. Int J Dermatol 52: 554-559, 2013.

13. Shainhouse T: Ocular manifestations of psoriasis. EC Ophthalmol 5: 172-176, 2017.

14. Maitray A, Bhandary AS, Shetty SB and Kundu G: Ocular manifestations in psoriasis: Original research article. Int J Ocul Oncol Oculoplasty 2: 123-131, 2016.

15. Jäger K, Garreis F, Posa A, Dunse M and Paulsen FP: Functional relationship between cationic amino acid transporters and beta-defensins: Implications for dry skin diseases and the dry eye. Ann Anat 192: 65-69, 2010.

16. Freeman AK, Linowski GJ, Brady C, Lind L, Vanveldhuisen P, Singer G and Lebwohl M: Tacrolimus ointment for the treatment of psoriasis on the face and intertriginous areas. J Am Acad Dermatol 48: 564-568, 2003.

17. Lee DJ: Intraocular implants for the treatment of autoimmune uveitis. J Funct Biomater 6: 650-666, 2015.

18. Durrani K and Foster CS: Psoriatic uveitis: A distinct clinical entity? Am J Ophthalmol 139: 106-111, 2005

19. Biggioggero $M$, Crotti $C$, Becciolini A, Miserocchi E and Favalli EG: The management of acute anterior uveitis complicating spondyloarthritis: Present and future. Biomed Res Int 2018: 9460187, 2018.

20. Dinu A, Bucur S, Olteanu R, Constantin T, Raducan A, Baetu M and Constantin MM: Psoriatic arthritis: A permanent new challenge for dermatologists (review). Exp Ther Med 20: 47-51, 2020

21. Vallet H, Seve P, Biard L, Baptiste Fraison J, Bielefeld P, Perard L, Bienvenu B, Abad S, Rigolet A, Deroux A, et al: Infliximab versus adalimumab in the treatment of refractory inflammatory uveitis: A multicenter study from the french uveitis network. Arthritis Rheumatol 68: 1522-1530, 2016.

22. Yang P, Zheng M, Zhang L, Du L, Zhou Q, Cai T, Qi J, Liang L and Kijlstra A: Uveitis in Chinese patients with psoriasis. Ocul Immunol Inflamm 25: 855-865, 2017.

23. Gladman DD: Clinical aspects of the spondyloarthropathies. Am J Med Sci 316: 234-238, 1998.

24. Ionescu RA: Uveitis as an extraarticular manifestation in spondyloarthritis. Rom J Rheumatol 29: 147-150, 2020.
25. Lee CY, Chen HC, Lin HW, Huang JY, Lin TL, Yang CH, Yeh CB, Lin HY and Yang SF: Increased risk of keratopathy after psoriasis: A nationwide population-based study. PLoS One 13: e0201285, 2018.

26. Lambert JR and Wright V: Eye inflammation in psoriatic arthritis. Ann Rheum Dis 35: 354-356, 1976.

27. Clarke SLN, Sen ES and Ramanan AV: Juvenile idiopathic arthritis-associated uveitis. Pediatr Rheumatol Online J 14: 27, 2016.

28. Fraga NA, Oliveira Mde F, Follador I, Rocha Bde O and Rêgo VR: Psoriasis and uveitis: A literature review. An Bras Dermatol 87: 877-883, 2012.

29. Dalvi SR, Yildirim R and Yazici Y: Behcet's syndrome. Drugs 72: 2223-2241, 2012

30. Pan J, Kapur M and McCallum R: Noninfectious immune-mediated uveitis and ocular inflammation. Curr Allerg Asthma Rep 14: 409, 2014.

31. Yanoff $\mathrm{M}$ and Cameron D: Diseases of the visual system. In: Goldman's Cecil Medicine. 24th edition. Goldman L and Schafer AI (eds). Elsevier Saunders, Philadelphia, PA, pp2426-2442, 2012.

32. Caspi RR: A look at autoimmunity and inflammation in the eye. J Clin Invest 120: 3073-3083, 2010.

33. Singla MB, Hodge DK and Moawad FJ: Infliximab-induced anterior uveitis in a patient with ulcerative colitis. J Crohns Colitis 9: 432, 2015.

34. Lee S, Park YJ and Lee JY: The effect of tumor necrosis factor-alpha inhibitors on uveitis in patients with ankylosing spondylitis. J Korean Med Sci 34: e278, 2019.

35. Yamaguchi K, Hayashi T, Takahashi G, Momose M, Asahina A and Nakano T: Successful certolizumab pegol treatment of chronic anterior uveitis associated with psoriasis vulgaris. Case Rep Ophthalmol 9: 499-503, 2018.

36. Lu J, Tang S, Yu N, YiX andLi Y: Successful secukinumab treatment of erythrodermic psoriasis and psoriatic arthritis concomitant with severe noninfectious uveitis: A case report. J Int Med Res: Nov 8, 2020 (Epub ahead of print). doi: 10.1177/0300060520969494

37. Mugheddu C, Atzori L, Del Piano M, Lappi A, Pau M, Murgia S, Zucca I and Rongioletti F: Successful ustekinumab treatment of noninfectious uveitis and concomitant severe psoriatic arthritis and plaque psoriasis. Dermatol Ther 30, 2017.

38. Benucci M, Damiani A, Li Gobbi F, Grossi V, Infantino M, Manfredi M, Niccoli L and Cantini F: Therapeutic potential of ixekizumab in the treatment of ankylosing spondylitis: A review on the emerging clinical data. Ther Clin Risk Manag 16: 287-297, 2020. 